\title{
Climate Change Assessment Using Negative Extreme Deviations of Surface Temperature Field (Case Study: Tbilisi)
}

\author{
Kukuri Tavartkiladze', Nana Bolashvili1, Maia Ananidze², Nikoloz Suknidze1, \\ Giorgi Chartolani 1 (ㅇ) \\ ${ }^{1}$ Vakhushti Bagrationi Institute of Geography, Tbilisi State University, Tbilisi, Georgia \\ ${ }^{2}$ Batumi Shota Rustaveli State University, Batumi, Georgia \\ Email:kuktav@mail.ru,nana.bolashvili@tsu.ge,nika.skt@gmail.com, giorgi.chartolani@tsu.ge
}

How to cite this paper: Tavartkiladze, K., Bolashvili, N., Ananidze, M., Suknidze, N. and Chartolani, G. (2020) Climate Change Assessment Using Negative Extreme Deviations of Surface Temperature Field (Case Study: Tbilisi). Open Journal of Geology, 10, 491-500.

https://doi.org/10.4236/ojg.2020.105021

Received: December 25, 2019

Accepted: May 9, 2020

Published: May 12, 2020

Copyright $\odot 2020$ by author(s) and Scientific Research Publishing Inc. This work is licensed under the Creative Commons Attribution International License (CC BY 4.0).

http://creativecommons.org/licenses/by/4.0/

(c) $\underset{\mathrm{By}}{\mathrm{i}}$ Open Access

\begin{abstract}
To conduct regional climate change assessment for the selected location (Tbilisi) more than century period hydrometeorological observation data of daynight minimal temperature is used. In the presented paper only temperature negative values of whole winter season are used. The temperature field change is characterized by the following four parameters: the minimal temperature sum (winter-day temperature is less than zero) of winter frost days; frost day number; season minimal temperature and the occurrence date of season minimal temperature. The modular structure of the above listed parameters has been studied and the mathematical expressions for temporal changes of those parameters have been obtained considering dynamical norms and cyclical changes. The intensity of this change in terms of global warming has been determined. One of the main parameters determining climate change is the variations of temperature field. Despite the fact that this parameter has been modified in the large range due to numerous impacts, they kept stable and provide sustainable equilibrium of the Earth's energy potential during long-term (several decades) period. This was the reason why the Earth climate remained unchained. As it is known, the anthropogenic impact on the environment has breached the sustainable balance of the Earth energy potential, the potential has been gradually grown almost for more than a century and is known by the name of global warming. The abovementioned process has already created the greatest threat to the existence of Earth's biosphere and if it still continues the catastrophic results eventually have to be expected. This led that the problem solving has been set as an urgent task. The numerous fundamental studies for all regions of the world have been carried out to assess the intensity of this process.
\end{abstract}




\section{Keywords}

Climate Change, Dynamic Norm, Negative Extreme Deviations, Surface Temperature

\section{Introduction}

In order to determine the intensity of the temperature field regional changes mainly the surface temperature regime multi-year actual data or so called norm variations are used. According to those variations to determine the intensity of change is complicated by the fact that the change tendency value is much smaller than the annual actual variations. The accuracy of the results will be increased if we use extreme deviations instead of the norm. However, the number of cases used is decreased that coarse the accuracy of the results obtained. It may be compensated by the increasing actual data period. Many attempts have been devoted to ascertaining climate change peculiarities [1]-[8] using temperature extreme variations as it is evident from modern scientific publications.

The present work has set forth an attempt to provide more reliable values of temperature field changes using negative extreme deviations, than the dynamic norm determines it by the average monthly or annual data [9] [10]. The research was conducted on the particular example, using Tbilisi meteorological station 1881-2012 year day-night negative, minimal temperature data. In particular, the only negative, minimum temperature values were chosen from eight values of measured temperature in every three hours per day. Because the negative, extremely low temperature in Tbilisi conditions are observed only in winter, the 90 day data have been used, from 1 December up to 28 February. The following procedures were performed for the purpose of preparing the primary data research: the 3 and 4 date day-night data was adjusted with 8-hour data; homogeneity was recovered, which was caused by the replacement of the meteorological station; some omitted observations were restored. These three procedures were carried out using the natural orthogonal disintegration method of random function in multidimensional space [11].

In addition, we carried out research not only to study negative extreme temperature regime structure, but also to increase the number of parameters that have contributed to the regime structure of the temperature field and the influence of global climate change on them is unknown. Such parameters are the following:

1) Seasonal sum of negative extreme temperature-sMT (namely 90 day-night negative, extreme deviations sum for our conditions);

2) The number of season frosty days, i.e. number of days when minimal day-night temperatures $\mathrm{nMT}<0$;

3) Season absolutely minimal temperature-mMT;

4) Corresponding date of season absolutely minimal temperature-tMT. 
Naturally suppose that these parameters must be changed in the disruption of Sun-Atmosphere-Earth sustainable equilibrium process. But how these changes are identical to each other and can they introduce additional common information to climate change assessment. This can be ascertained by identification of their mutual correlation. For this purpose, the autocorrelation matrix of the introduced parameters has been built, which determines both the mutual and correlative connections with the main characteristic parameter of the temperature field - the average season day-night temperature (avT). It is given in Table 1.

As it is evident from Table 1 these parameters have different correlation between each other with the season average day-night temperature, except for the corresponding absolutely minimum season temperature date and therefore have independent information that can be used to evaluate the intensity of temperature field changing.

It has already been mentioned that the solution of the proposed task is carried out on the particular example, on Tbilisi Meteorological station data, which has a longest observation period in the history of meteorological observations. The assessment of temperature field change was carried out in order of climate change general assessment in the winter season using differential and linear approximation.

The discussed period of the winter season we divided into three parts to evaluate temperature field changes by so called differential method. The changing process has been evaluated by the average temperature differences of third and first periods of the season. This difference, along with the average temperature of the entire period, is given in Table 2. The table shows that the average day-night temperature of the winter season increases by $0.59^{\circ} \mathrm{C} / 100$ year intensity. It is noteworthy that, compared to the standard (stable) norm, the variable norm obtained from the differences reduces deviation by $24.2 \%$.

Table 1. Autocorrelation matrix of determining parameters of winter season day-night minimal temperature modular structure (according to 1881-2012 Tbilisi actual data).

\begin{tabular}{cccccc}
\hline & avT & sMT & NMT & mMT & tmMT \\
\hline avT & 1 & 0.887 & -0.855 & 0.774 & -0.042 \\
sMT & 1 & -0.883 & 0.801 & 0.061 \\
NMT & & 1 & -0.67 & 0.023 \\
mMT & & & 1 & 0.083 \\
tmMT & & & & 1
\end{tabular}

Table 2. 1881-82: 2011-12 winter season temperature change in Tbilisi by initial and last 44 year period mean temperature differences.

\begin{tabular}{ccccc}
\hline \multirow{2}{*}{ Winter season periods } & $\begin{array}{l}1881-82 \\
2011-12\end{array}$ & $\begin{array}{c}1881-82 \\
1923-24\end{array}$ & $\begin{array}{c}1968-69 \\
2011-12\end{array}$ & $\begin{array}{c}\text { Changes during } \\
100 \text { years }\end{array}$ \\
\cline { 2 - 5 } & & & & \\
\hline Day-night mean temperature $\left({ }^{\circ} \mathrm{C}\right)$ & 2.44 & 2.09 & 2.74 & 0.74 \\
\hline
\end{tabular}


Temperature field change may be accurately identified by linear approximation of given temperature series. Such approximation has been conducted and line resulted from least square method [12] is given in Figure 1.

On the horizontal and vertical axes of the drawing, the average temperature and year of the season are adjusted. The drawn line defines the dynamic norm, which is expressed as follows:

$$
\operatorname{avT}=1.68+0.0116 \times \tau,
$$

where, $\tau$ is year sequence from 1881 . Thus temperature change intensity obtained by linear approximation is $1.16^{\circ} \mathrm{C} / 100$ year.

The regime structure of the all introduced parameters is determined by the same way. In particular, the linear and possible cycle changes were considered in the mathematical expression of the parameter temporal variation. The relevant expression has obtained the following type:

$$
X=a \tau+b+A * \operatorname{Sin}(2 \pi \tau / L+F),
$$

where empirical members characterizing linear $(a, b)$ and cyclical $(A, L, F)$ processes determine the parameter $(X)$ special variation. Their values are defined by the least square and iteration methods correspondingly. The obtained value has been considered reliable if the standard deviation of the outcome of the expression (2) was less than the standard deviation of dynamic norm of the same process.

\section{Seasonal Sum of Negative Temperature (sMT)}

The extreme deviations sum of only winter day-night temperature is one of the main characteristic of climate change. After determining the relevant empirical coefficients, the Equation (2) has been transformed into the type:

$$
\mathrm{sMT}=-0.96 \tau-235.14+32.0 \times \operatorname{Sin}(2 \pi \tau / 22.1+2.8) .
$$

The actual values of winter season day-night temperature negative deviation sums are given in Figure 2. Also, there are presented calculated values of Equation (3).

How it is appropriate to express the temperature temporal change by equation (3) can be ascertained by comparison of the standard error of equation result with result error of dynamic norm. We performed appropriate calculations and it turned out that the results of dynamic norms specify the results obtained with statistical norms by $8.8 \%$ and the result of Equation (3) is more accurate with $4.0 \%$ than the result obtained by dynamic norms.

\section{The Number of Frosty Days (nMT)}

The number of frosty days (nMT) is a significant characteristic of the winter season's regime. The sum of the winter day-night only negative temperature extreme deviations may be the same between two seasons, while the frosty day's number varies greatly. Therefore, in the background of global warming, its regional change identification has great significance. 


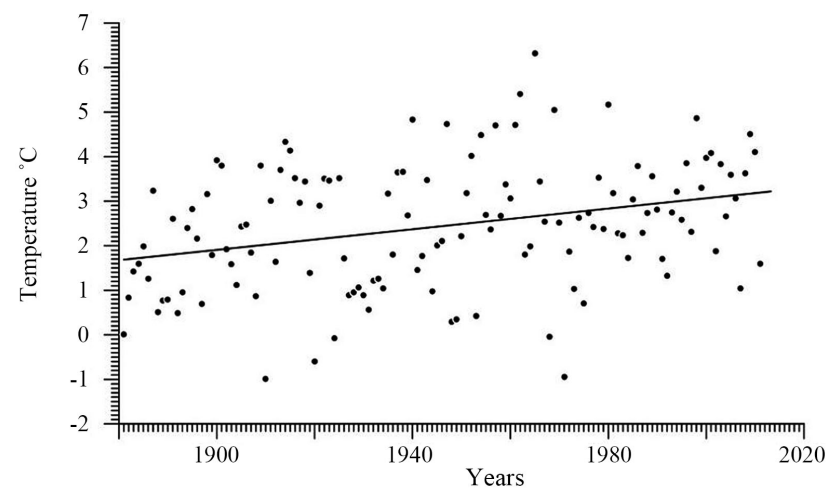

Figure 1. Day-night mean temperature values of 1881-82; 2011-12 winter seasons and its linear approximation.

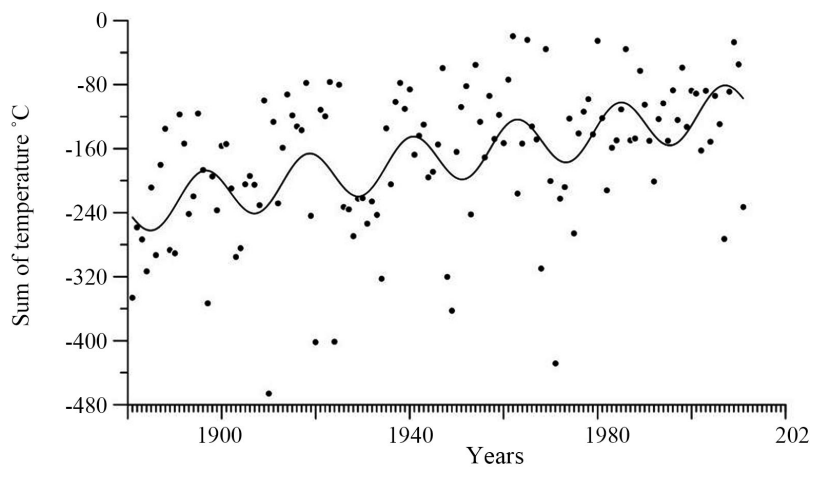

Figure 2. Values of day-night temperature extreme deviations and their calculated ones by Equation (3) according to the 1881-2012 winter season of Tbilisi.

We used the available actual data to determine the number of frosty days of each season and their change according to the season presented by the equation (2). It is transformed into the following after calculation of empirical coefficients:

$$
\mathrm{nMT}=-0.153 \tau-60.78+1.58 \times \operatorname{Sin}(2 \pi \tau / 21.5+2.9) .
$$

Using the obtained results the Figure 3 has been drowned, which shows the number of frosty days according to years and their variation according to the Equation (4).

As it is evident from Figure 3, the number of frosty days seems to have a significant decline. For 131 years, the minimal frost day of the season (90 days) was amounted to 81 and it was recorded in the winter of 1965-1966. The minimal frost days were fixed in winter of 1920-1921, and covered only 13 days, while the average amount of frosty days was 50 of discussed seasons.

The changing intensity of frozen days determined by dynamic norms is 15.3 days/100years. This specifies the calculated values by $24.7 \%$ compared with statistical norms. The approximation with the actual regime considering the cyclical process is less effective as it specifies the results of dynamic norms by only $0.2 \%$. It is also evident from this drawing: the curve drowned by equation almost approaches the line due to the small values of amplitude. 


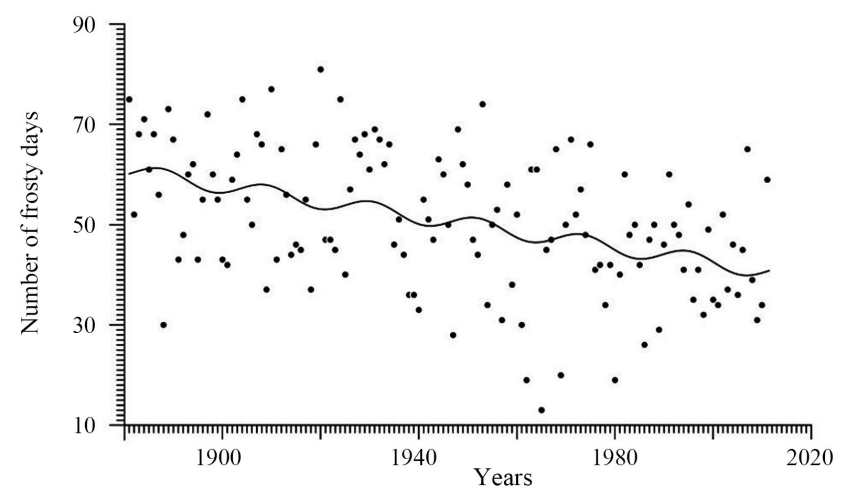

Figure 3. Winter season frosty day number during 1881-2012 period in Tbilisi and their changing calculated by Equation (4) according to the years.

\section{The Absolute Minimum Temperature of the Winter Season (mMt)}

One of the characteristic parameter of the negative extreme deviation of the winter season temperature field is the season absolutely minimal temperature, i.e. the extremely minimal temperature value from the actual observation series. The absolute minimum temperature values of each season and their change according to the Equation (2) in 1881-2012 are given in Figure 4.

As the figure shows, the absolute minimal temperature change range is quite large. In particular, it was observed from $-20.5^{\circ} \mathrm{C}$ to $-2.7^{\circ} \mathrm{C}$, in morning hours of 29 of January, 1881 and 19 of January, 1962 correspondingly.

Under Tbilisi conditions the winter season minimal temperature change is characterized by increasing tendency of $3.33^{\circ} \mathrm{C} / 100$ year. The temperature value determined by dynamic norms increases the accuracy by $8.0 \%$ compared to statistical norms. And the consideration of cyclical change, the optimal value of which is determined by the following equation specifies by $3.5 \%$ the approximation defined from the dynamic norm.

$$
\mathrm{mMT}=-0.0333 \tau-11.87+1.10 \times \operatorname{Sin}(2 \pi \tau / 22.1+3.8) .
$$

It is noteworthy that the variations of extreme values characterize the atmosphere processes with better approximation than the day-night average temperature. This once again is confirmed by the fact that to fix the cyclic change in temperature field variations in the atmosphere is a difficult process. The different parameters determining extreme temperature field gave us similar results, taking into consideration the entirely objective, independently conducted analysis and the error limits.

\section{The Exact Date of the Absolute Minimal Temperature (tMT)}

Yet from Table 1, it seemed that the season's absolutely minimal temperature appropriate date variations have no connection with the variations of extreme temperature characterizing parameters. And this clearly demonstrates that the 
global warming process would not influence it. The extreme temperature actual data which is used in this paper allows examining this fact. After determining the distance (days) of season absolutely minimal temperature date since December 1, on the obtained series we performed the same procedures that were used on other parameters. Variations of the members of the received series according to the season change and their approximation by Equation (2) is given in Figure 5.

As it is evident from the drawing, the change range of the absolute minimal temperature date covers the whole season. And the temporal change curve has no decrease or increase tend. The possible cyclic change is weakly revealed. It is noteworthy that the cycle period is $\mathrm{L}=20.2$ years from the truly objectively defined cycle parameters.

As it is known, due to the difference between the Earth day-night rotation around the axis and the difference between the calendar times, the inter-annual temperature regime has been shifted. It is necessary several century actual data to completely examine this. Because the actual data of extremely negative temperature used in this paper covers more than a century, we have tried to see if the database used by us accounts for a seasonal shift. For this, we divided winter season data in two periods: 1881/82-1946/47 and 1947/48-2011/12.

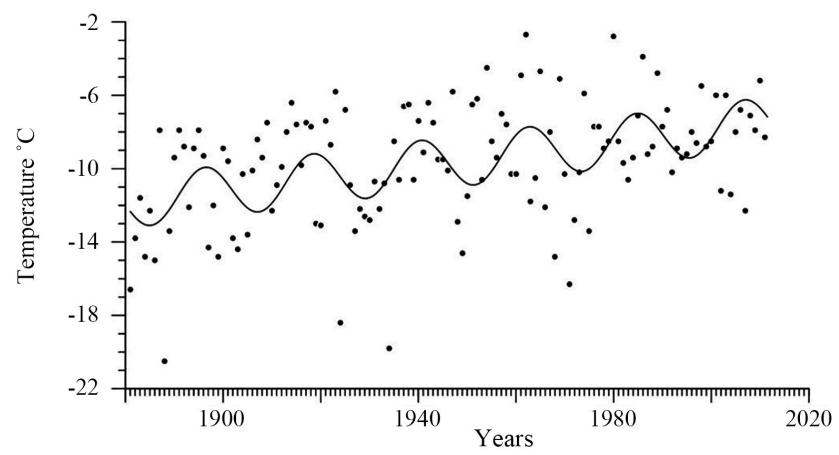

Figure 4. The absolute minimal temperature values in winter season of 1881-2012 and their change calculated by Equation (5) according to the years.

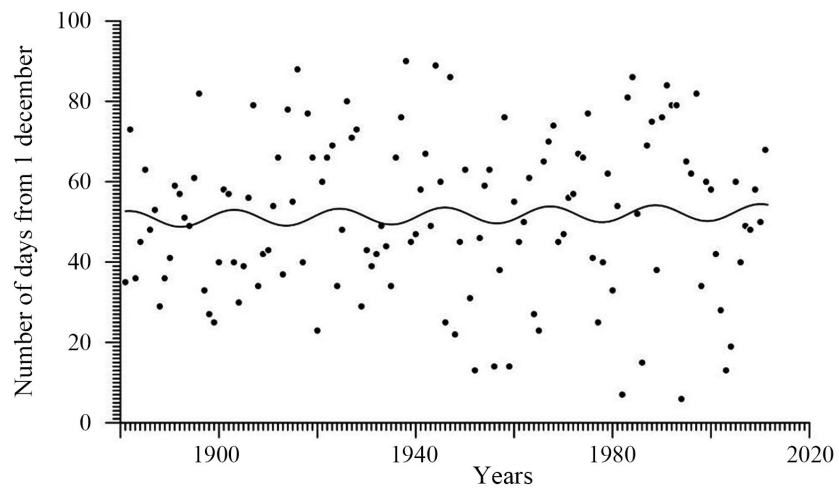

Figure 5. The dates of absolute minimal temperature of the winter season (number of days from December 1) according to the season and their approximation with regard to cycling. 
We determined the seasonal change of day-night negative minimal temperature for each period. Comparison of the relevant dates of the two seasonal minimal values would be allowed us to determine the season's immutability or shift values.

It is noteworthy that the temperature field forming processes are affected by the numerous processes in the atmosphere, causing accidental deviations which are hardly predictable. Therefore, in order to exclude random deviations, we took no actual data, but optimally (by the least square method) obtained curves. The derived curves are presented in Figure 6.

Replacement of relevant days of the minimum values of the curves determines the seasonal shift. As it is evident from the drawing the shift approximately amounts 7 days. In particular, if in the first period the probabliest date of the absolute minimum is January 17, in the second period it will be moved by 7 days and reaches 24 January. The fact is that this result quite exceeds the expected shift, but according to the existed observations, it is very difficult to determine the reason. As for the regional climate change value or the temperature difference between the minimum of two periods is $3.3^{\circ} \mathrm{C} / 100$ year in the conditions of Tbilisi.

\section{The Following Recommendation May Be Issued Based on Conducted Research}

1) The impact of global climate warming on the regional one is better to evaluate using negative extreme deviation of winter season temperature instead of annual temperature series. It requires increasing of research period but must significantly improve approach accuracy with actual change. It is desirable to study not only temperature negative extreme deviations but also investigate the following parameters of temperature field: daily minimal, negative, negative temperature seasonal sum, winter season frosty (when temperature is below zero) day number, season absolute minimal, date of absolute minimal temperature.

2) The autocorrelation matrix constructed with mentioned parameters must confirm that the parameters aren't identical towards each other, i.e. each one gives some information different from other parameters.

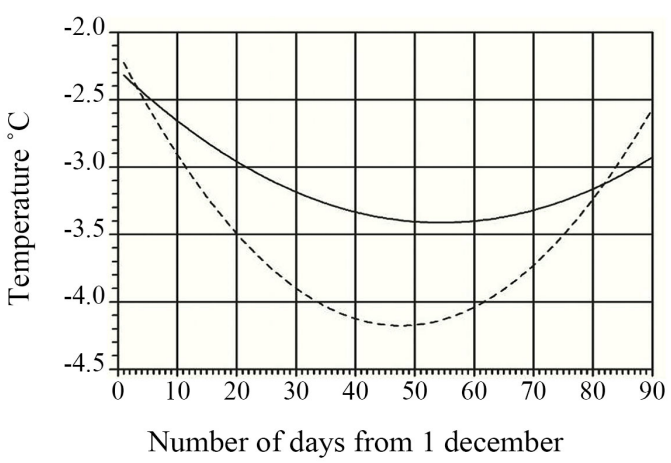

Figure 6. Daily minimal negative temperature distribution 1881/82-1946/47 (intermittent curve) and 1947/48-2011/12 by winter season. 
3) The regime structure of those parameters or parameters temporal change regularity in given period must be evaluated by using the same method. In particular parameters random variations must be characterized by dynamical norm considering cyclical changes.

\section{The Novelty of Research and Conclusion}

The novelty of presented research is that firstly climate change assessment has been carried out on Tbilisi case study using temperature field negative extreme deviations. Based on obtained results the following may be concluded:

1) The minimal (negative) temperature seasonal sum obtained from difference method has been reduced by $3.8^{\circ} \mathrm{C} / 100$ year compared with statistical norm or norm that doesn't reflect changes at all, which is about $3 \%$ of the multiyear average value of this parameter. The same change calculated by dynamical norm approached true value by $8.8 \%$ in comparison with statistical norm. And quantity calculated with cyclical change improves value defined by dynamical norm by $4.0 \%$;

2) Considering cyclical changes the frosty day number has been reduced by 15.3 day/100year, this value improves the one defined from dynamic norms by $7.8 \%$;

3) Absolute minimal temperature has been increased by $3.3^{\circ} \mathrm{C} / 100$ year. The dynamic norm specifies the result by $3.5 \%$ compared to static norm. The obtained result improves the result obtained from dynamical one by $3.6 \%$ taking into account cyclical changes;

4) The exact date of the corresponding absolute minimum does not have change tendency, the temperature increases by $3.3^{\circ} \mathrm{C} / 100$ year, only its interseasonal shifting from 17 to 24 January takes place. This shift should not have any connection with climate change; it should be caused by the astronomical factor.

\section{Conflicts of Interest}

The authors declare no conflicts of interest regarding the publication of this paper.

\section{References}

[1] Tavartkiladze, K., Begalishvili, N., Tsintsadze, T. and Kikava, A. (2012) Influence of Global Warming on the Near Surface Air Temperature Field in Georgia. Bulletin of the Georgian National Academy of Sciences, 6, 55-60.

[2] Khlebnikova, E.I., Rudakova, Y.L., Sall, I.A., Efimov, S.V. and Shkolnik, I.M. (2019) Changes in the Extremes of Thermal Regime in the XXI Century: Ensemble Estimates for the Territory of Russia. Meteorology and Hydrology, 3, 11-23. https://doi.org/10.3103/S1068373919030014

[3] Gruza, G.V. and Rankova, E.Y. (2009) Climate Change Detection: State, Variability and Extremes of Climate. Meteorology and Hydrology, 11, 15-29. 
[4] Kiktev, D.V., Size, D. and Alexander, L. (2009) Comparison of Multiyear Averages and Trends in Annual Extremes of Temperature and Precipitation According to Simulation and Observation Data. Bulletin of RAS: Physics of Atmosphere and Ocean, 45, 305-315.

[5] Platova, T.V. (2007) Climatic Characteristics of Some Indicators of Surface Air Temperature and Precipitation Extremes in Russia. Bulletin "Use and Protection of natural Resources in Russia". Publishing House NIA-Nature, 38-47.

[6] Bulygina, O.N., Razuvaev, V.N., Korshunova, N.N. and Groisman P.Y. (2007) Climate Variations and Changes in Extreme Climate Events in Russia. Environmental Research Letters, 2, 045020. https://doi.org/10.1088/1748-9326/2/4/045020

[7] Thompson, D.W.J. and Wallace, J.M. (1998) The Arctic Oscillation Signature in the Wintertime Geopotential Height and Temperature Fields. Geophysical Research Letters, 25, 1297-1300. https://doi.org/10.1029/98GL00950

[8] Karl, T., Nicholls, N. and Ghazi, A. (1999) Weather and Climate Extremes: Changes, Variations and a Perspective from the Insurance Industry. WCE. Kluwer Academic Publishers, 349 p.

[9] Tavartkiladze, K., Qiqava, A. and Solomonidze, R. (2011) Surface Layer Temperature Field Forecasting on Adjara Territory until 2030. Khandzta, 4, 67-78.

[10] Gruza, G.V. and Rankova, E.Y. (2012) Dynamical Climatic Norms of Air Temperature. Russian Meteorology and Hydrology, 37, 717-727. https://doi.org/10.3103/S1068373912110052

[11] Obukhov, A.M. (1960) On Statistical Orthogonal Decomposition of Empirical Functions. Bulletin of Academy of Sciences of the USSR. Geophysics, 3, 432-439.

[12] Mazmishvili, A.I. (1968) The Least Square Method. Nedra, Moscow, 436. 